\title{
Summary of the 12th International Workshop on Models@run.time
}

\author{
Sebastian Götz*, Nelly Bencomo ${ }^{\dagger}$, Kirstie Bellmann ${ }^{\ddagger}$, Gordon Blair ${ }^{\S}$, \\ *Technische Universität Dresden, Germany, Email: sebastian.goetz@acm.org \\ ${ }^{\dagger}$ Aston University, UK, Email: nelly@acm.org \\ ‡Topcy House Consulting, US, Email: bellmanhome@yahoo.com \\ $\S$ Lancaster University, UK, Email: gordon@comp.lancs.ac.uk
}

\begin{abstract}
This year the 12th edition of the workshop Models@run.time was held at the 20th International Conference on Model Driven Engineering Languages and Systems. The workshop took place in the city of Austin, Texas, USA, on the 18th of September 2017. The workshop was organized by Sebastian Götz, Nelly Bencomo, Kirstie Bellman and Gordon Blair. Here, we present a summary of the workshop and a synopsis of the topics discussed and highlighted during the workshop.
\end{abstract}

\section{INTRODUCTION}

Once more, the models@run.time community [2], [3], [5] has met at the MODELS conference and discussed the newest early stage research in our field. Compared with previous editions of workshops in MODELS, in general, the number of submissions dropped and it included our workshop. Notably, this did not have any impact on the number of participants at the workshop which was quiet high again. Although this year, only 4 papers were accepted for presentation at the workshop, we had approximately 20 active participants throughout the day and lots of lively discussions. Table I and Figure 1 show how the workshop quantitatively evolved over the last years.

\begin{tabular}{|c||c|c|c|}
\hline Year & Attendees & Submissions & Accepted (Long+Short) \\
\hline 2008 & 44 & 20 & $6+6$ \\
2009 & 49 & 16 & $4+2$ \\
2010 & 35 & 15 & $4+6$ \\
2011 & 26 & 10 & $4+2$ \\
2012 & 48 & 18 & 11 \\
2013 & 25 & 20 & $7+2$ \\
2014 & 27 & 8 & 5 \\
2015 & 36 & 13 & 8 \\
2016 & 30 & 12 & 7 \\
2017 & 20 & 7 & 4 \\
\hline
\end{tabular}

KEY NUMBERS OF THE MRT WORKSHOP SERIES

\section{WORKSHOP SESSIONS}

Among the participants, there were many new researchers an observation we regularly have over the last years. Hence, we started the workshop with an introduction to models@run.time in three parts. First, Nelly Bencomo gave a historical perspective on the last 12 years of models@run.time based on [1]. After, Sebastian Götz showed first results from an upcoming literature survey on models@run.time covering 271 papers from the field. Finally, Kirstie Bellman rounded up the introduction by teasing challenging problems to be solved in the

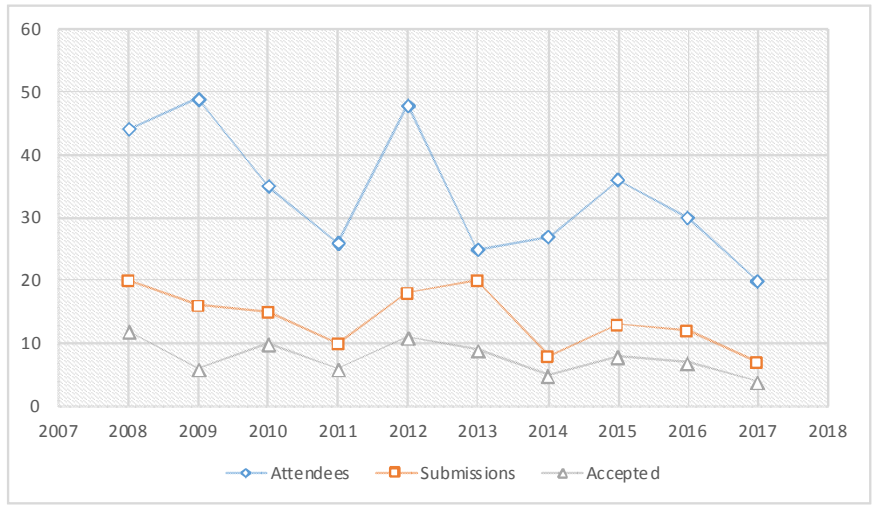

Fig. 1. The MRT Workshop Series since 2008

future by the community. After this introduction, two sessions with paper presentations followed. The last session was used for an intensive discussion of what the community considers as challenging problems to be solved. In the following, all four sessions will be summarized in more detail.

\section{A.Models@run.time for Evolution}

The first session covering presentations by the authors focused on the application of models@ run.time with the purpose to support software evolution.

Hassan Gomaa gave the first talk, presenting his paper, which was jointly authored with Emad Albassam, with the topic "Run-time Software Architectural Models for Adaptation, Recovery and Evolution". He presented an overview of architectural runtime models and their use for adaptation recovery as well as evolution.

The second talk was given by Dhaminda Abeywickrama, who presented his paper, jointly authored with Eila Ovaska, with the topic "Reflexive and Evolutional Digital Service Ecosystems with Models at Runtime". In contrast to the first paper, his approach applied models@run.time at three abstraction levels: at the level of goals, of architecture and of the domain/context. The approach was evaluated using a case study from the ambient assisted living domain.

\section{B. Models@run.time for Assurance}

The second session covering paper presentations had assurance as its theme. 
Byron Devries gave the first presentation on his paper, jointly authored with Betty Cheng, having the title "Using Models at Run Time to Detect Incomplete and Inconsistent Requirements". He presented an approach to detect incomplete requirements at runtime. Thus, this approach applied models@run.time at the abstraction level of requirements. The approach was evaluated using a case study from adaptive cruise control.

The second talk was given by Christopher Landauer on his paper entitled "Active System Integrity Fences". Fences, as introduced by Christopher Landauer, are a generalized concept of contract-based programming, i.e., the application of modelbased contracts at system runtime. The approach was evaluated argumentatively in the context of safety-critical systems.

\section{Beyond Models@run.time}

Several issues were discussed during the last 60 minutes of the workshop.

One issue discussed was the need for a definition of models@run.time-based architectural patterns, which was coined by Peter Clarke. The idea is to take advantage of the experience of the construction of different architectures during more than a decade and to categorise the different experiences by using patterns. By this, we could start discussing models@run.time with the software architecture community.

There was also discussion about using the notion of transactions to support the causal connection needed to fully support runtime models. The need to do further research on the use of machine learning techniques for emerging runtime models was also part of the lively discussion. Finally, Kirstie Bellman introduced the idea of using runtime models for $\mathrm{V} \& \mathrm{~V}$ at runtime.

In the end, the audience agreed that it is a good idea to run the workshop again in 2018.

\section{CONCLUSION}

The twelfth edition of the international workshop on models@run.time was again very well visited (20 participants).

The trend of submissions was low in comparison to the last years. Notably, although only few papers were submitted (5), as a set they presented high quality, which allowed us to accept four (4) papers.

One of the defining characteristics of the MRT workshop is a commitment to discussion time and this year's workshop continued that tradition in a lively and informative fashion. In order to develop high quality models for use at runtime, we need to deal with the classical AI knowledge engineering problem of how to get the knowledge that is in an experts head OR in the systems sensors and status databases into the models. One of the challenges going forward is to capture the knowledge that is meaningful to the tasks and the decisions of the system; this requires a great deal of flexibility and metaknowledge in the reasoning processes.

Another topic that was widely discussed was the need for self-explanation so that the human users and developers could better monitor when and how the different runtime models were being used. This was noted as being especially important when the system has crashed and has switched to new configurations, and for assessing the progress towards new goals. In a separate discussion, there was a great deal of interest by the workshop participants in the growing literature review, comparison, and gap analysis being done on 271 papers on Models@run.time by Götz and Bencomo. This led to a great deal of discussion on how to characterize the diverse findings and what to do with the information gained from the study and especially how to encourage filling in the research gapsby the community.

Branching off from this discussion,we also discussed how to integrate multiple runtime models.

We ended the workshop with a discussion of some of the overarching challenges and problems for MRT.

This included technical challenges and social challenges for identifying and bringing in the expertise we need into the community. Among the technical challenges, one keychallenge was to better define and support the relationship between design time and MRT systems for long-term improvement, analysis, and system understanding. Another was the importance of discovery processes for the ability to find the relevant MRT models for a given goal, especially in distributed systems. The third major challenge was how to share results (including how to gauge their relevancy) among different MRT models in distributed, complex systems.

Among the social issues, we discussed how to encourage the community to develop more theoretical foundations for such difficult MRT issues as integration, self-modeling, and model generation. We also discussed what kinds of new expertise we need in the community to better address MRT challenges.

Since the first edition of the workshop, it was constantly co-located to the MODELS conference and, consequently, mainly attracted participants from the modelling community. However, this year for the second time we also ran a separate edition of models@run.time at ICAC [4] to attract people from self-aware and autonomous computing. We plan to continue both editions next year, i.e., a third workshop on models@run.time for self-aware computing systems at ICAC and a 13th workshop on models@run.time at MODELS.

\section{REFERENCES}

[1] Nelly Bencomo. The role of models@run.time in autonomic systems: Keynote. In 2017 IEEE International Conference on Autonomic Computing, ICAC 2017, Columbus, OH, USA, July 17-21, 2017, pages 293-294, 2017.

[2] Nelly Bencomo, Robert France, Betty H. C. Cheng, and Uwe Aßmann, editors. Models@run.time. Foundations, Applications, and Roadmaps, volume 8378. Springer LNCS, 2014.

[3] G. Blair, N. Bencomo, and R.B. France. Models@run.time. Computer, 42(10):22-27, Oct 2009.

[4] Sebastian Götz, Nelly Bencomo, Kirstie Bellman, and Gordon Blair. 1st international workshop on models@run.time for self-aware computing systems. In IEEE International Conference on Autonomic Computing (ICAC), pages 363-363, July 2016.

[5] Sebastian Götz, Nelly Bencomo, and Robert France. Devising the future of the models@run.time workshop. SIGSOFT Software Engineering Notes, 40(1):26-29, January 2015. 